\title{
Targeting the innate immune response with improved vaccine adjuvants
}

\author{
Achal Pashine, Nicholas M Valiante \& Jeffrey B Ulmer
}

Despite two centuries of vaccine use, only a few adjuvants and delivery systems are licensed for human use. This is partly because traditional vaccines based on attenuated live organisms already have them-their invasiveness provides efficient delivery to antigen-presenting cells and various naturally occurring components of the pathogens stimulate the innate immune system. But consideration of these immune potentiators and delivery systems has become important to the development of new subunit vaccines consisting of isolated antigens. Here we consider rational approaches to the discovery and development of immunostimulatory compounds and vaccine formulations that target innate immune responses.

\section{Vaccines}

Vaccines are considered by many to be one of most successful medical interventions against infectious disease ${ }^{1}$. But many significant obstacles remain, such as improving suboptimal vaccines, developing new ones against diseases for which no vaccines yet exist and responding rapidly to newly emerging pathogens ${ }^{2}$. In designing effective vaccines, several key elements are required (Box 1). First, an antigen is required, against which a memory immune response is targeted. Important considerations for antigens are their selection (particu-

larly in cases where there may be many to choose from, such as for bacterial pathogens) and presentation (for example, where tertiary structure must be preserved, such as for the induction of conformationspecific functional antibodies). Second, stimulation of the innate immune system is now known to have an important role in the evolution of the adaptive immune response ${ }^{3}$. Thus, inclusion of immune potentiators (also termed adjuvants), which trigger early innate immune responses to aid in the generation of robust and long-lasting adaptive immune responses, is crucial to vaccine effectiveness. Finally, delivery systems that target the vaccine (both antigen and immune potentiators) to appropriate cells of the immune system will ensure optimal stimulation ${ }^{4}$.

\section{Innate immune responses}

The immune system has evolved two main functions: to react quickly (within minutes) to molecular patterns found in microbes, and to develop slowly (over days to weeks), precisely targeted specific adaptive

Achal Pashine, Nicholas M. Valiante and Jeffrey B. Ulmer are at the Chiron Corporation, Emeryville, California 94608, USA. Correspondence should be addressed to J.B.U. (jeffrey_ulmer@chiron.com).

Published online 5 April 2005; doi:10.1038/nm1210

immune responses. The faster-acting innate immune responses provide a necessary first line of defense because of the relatively slow nature of adaptive immunity ${ }^{5}$. In contrast, adaptive immunity uses selection and clonal expansion of immune cells harboring made-to-order somatically rearranged receptor genes ( $\mathrm{T}$ - and $\mathrm{B}$-cell receptors) recognizing antigens from the pathogen, thereby providing specificity and longlasting immunological memory ${ }^{6}$. Innate immune responses, among their many effects, lead to a rapid burst of inflammatory cytokines and activation of antigen-presenting cells (APCs) such as macrophages and dendritic cells. These nonclonal responses also lead to a conditioning of the immune system for subsequent development of specific adaptive immune responses.

To distinguish pathogens from self-components, the innate immune system uses a wide variety of relatively invariable receptors that detect evolutionarily conserved signatures from pathogens (pathogen-

\section{BOX 1 VACCINE COMPONENTS}

Key elements of effective vaccines include the antigen, against which adaptive immune responses are elicited; immune potentiators to stimulate the innate immune system; and delivery systems to ensure that the vaccine is delivered to the right place at the right time. The main types of antigens, immune potentiators and delivery systems are listed below.

\section{Antigens}

- Whole inactivated or attenuated organisms or a mixture of various strains.

- Isolated and purified proteins, glycoproteins and carbohydrates

- Recombinant proteins and glycoproteins

Immune potentiators

- Bacterial products

- Toxins and lipids

- Nucleic acids

- Peptidoglycans

- Carbohydrates, peptides

- Cytokines and hormones

- Small molecules

Delivery systems

- Mineral salts

- Surface active agents

- Synthetic microparticles

- Oil-in-water emulsions

- Liposomes 


\section{PERSPECTIVE}

associated molecular patterns, $\mathrm{PAMPs}^{7,8}$ ). The addition of such microbial components to experimental vaccines leads to the development of robust and durable adaptive immune responses. The mechanism behind this potentiation of immune responses was not well understood until recently, when some of the pattern-recognition receptors (PRRs) involved in the innate immune responses to PAMPs were identified (Table 1). PRRs are differentially expressed on a wide variety of immune cells, including neutrophils, macrophages, dendritic cells, natural killer cells, B cells and some nonimmune cells such as epithelial and ๖ endothelial cells. Engagement of PRRs leads to the activation of some of these cells and their secretion of cytokines and chemokines, as well as maturation and migration of other cells. In tandem, this creates an inflammatory environment that leads to the establishment of the adaptive immune response ${ }^{9}$.

PRRs consist of nonphagocytic receptors, such as Toll-like receptors (TLRs) and nucleotide-binding oligomerization domain (NOD) proteins, and receptors that induce phagocytosis, such as scavenger receptors, mannose receptors and $\beta$-glucan receptors ${ }^{10}$. The latter receptors directly recognize ligands on the surface of pathogenic microbes and lead to their engulfment into phagocytic cells such as macrophages. Nonphagocytic receptors that recognize PAMPs extracellularly (certain TLRs) or intracellularly (NOD family of proteins) lead to an elaborate signal transduction cascade. Although reports suggest that some TLRs bind PAMPs directly ${ }^{11-13}$, it is not yet known whether this is generally true or whether intermediate protein components are involved.

\section{Table 1 Pattern-recognition receptors ${ }^{14}$}

\begin{tabular}{|c|c|}
\hline Pattern-recognition receptors & Ligands \\
\hline TLR1 $1^{a}$ & $\begin{array}{l}\text { Bacterial lipoproteins from Mycobacteria, } \\
\text { Neisseria }\end{array}$ \\
\hline TLR2 $^{\mathrm{a}}$ & $\begin{array}{l}\text { Zymosan yeast particles, peptidoglycan, } \\
\text { lipoproteins, glycolipids, } \\
\text { lipopolysaccharide }\end{array}$ \\
\hline TLR3 & Viral double-stranded RNA, poly:IC \\
\hline TLR4 & $\begin{array}{l}\text { Bacterial lipopolysaccharides, plant } \\
\text { product taxol }\end{array}$ \\
\hline TLR5 & Bacterial flagellins \\
\hline TLR6 ${ }^{a}$ & $\begin{array}{l}\text { Yeast zymosan particles, lipotechoic acid, } \\
\text { lipopeptides from mycoplasma }\end{array}$ \\
\hline TLR7 & $\begin{array}{l}\text { Single-stranded RNA, R- } 837 \text { and R848 } \\
\text { other synthetic compounds such as } \\
\text { loxoribine and bropirimine }\end{array}$ \\
\hline TLR8 ${ }^{c}$ & Single-stranded RNA, R848 \\
\hline TLR9d & CpG oligonucleotides \\
\hline TLR10 & Unknown \\
\hline TLR11 & $\begin{array}{l}\text { Bacterial components from uropathogenic } \\
\text { bacteria }\end{array}$ \\
\hline NOD $1, \mathrm{NOD}^{\mathrm{e}}$ & Peptidoglycans \\
\hline Scavenger receptors ${ }^{f}$ & $\begin{array}{l}\text { Acetylated/malelylated proteins; modified } \\
\text { low-density lipoproteins and other } \\
\text { polyanionic ligands }\end{array}$ \\
\hline $\begin{array}{l}\text { Macrophage mannose } \\
\text { receptors and other c-type } \\
\text { lectin receptors }\end{array}$ & $\begin{array}{l}\text { Sulfated sugars, mannose-, fucose- and } \\
\text { galactose-modified polysaccharides and } \\
\text { proteins }\end{array}$ \\
\hline Type 3 complement receptors & Zymosan particles, $\beta$-glucan \\
\hline
\end{tabular}

\section{and dectin type receptors}

aTLRs can form heterodimers, which further changes their specificity. For example, TLR2 anRs can form heterodimers, which further changes their specificity. For example, TLR2 have similarly been shown to cooperatively recognize a mycobacterial lipoprotein ${ }^{45}$. bR-837 (imiquimod) and R-848 (resiquimod) are the first small-molecule synthetic TLR ligands to be identified $^{30}$. R-837 is licensed for use as a topical cream (Aldara) against anogenital warts caused by human papilloma virus. ${ }^{\mathrm{T}} \mathrm{TLR} 8$ is suggested to be nonfunctional in mice ${ }^{46}$. ${ }^{\mathrm{d}} \mathrm{TLR} 9$ from human and mouse shows CpG ODN sequence specificity ${ }^{47}$. eNucleotide oligomerizing domain protein. Naturally occurring mutations in human have been shown to profoundly alter responsiveness to bacterial infections. 'Scavenger receptors are further subdivided into SR-A, SR-B, SR-D and SR-F depending on structure and ligand recognition ${ }^{10}$.
Signaling through TLRs and other PRRs has been reviewed recently in great detail ${ }^{14}$, thus will be only briefly discussed here. TLRs are type I integral membrane glycoproteins with considerable cytoplasmic domain homology to interleukin (IL)-1 receptors. A conserved cytoplasmic domain $\sim 200$ amino acids long, known as TIR (Toll-IL-1 receptor domain), is important for its association with adaptor proteins, thus linking these transmembrane receptors to their downstream signaling pathways. All TLRs, except TLR3, share a common signaling pathway that uses an adapter protein called MyD88 (myeloid differentiation factor 88). In some cases (for example, TLR5, TLR7, TLR9) MyD88 has been shown to bind directly by means of the TIR domain, whereas in others, (for example, TLR2, TLR4) another intermediate adapter molecule known as TIRAP (TIR domain-containing adapter protein) is involved. Through its amino-terminal death domain, MyD88 binds to another family of signal transducers, known as IRAKs (IL-1 receptorassociated kinases), which in turn bind to TRAF6. Subsequent activation of the transcription factors NF- $\mathrm{KB}$ and AP-1 by TRAF6 involves yet other adaptor proteins known as TAK1 and TAB2 (refs 14,15; Fig. 1).

NOD proteins are involved in intracellular recognition of pathogens and their products such as muramyl dipeptide peptidoglycans from bacteria.

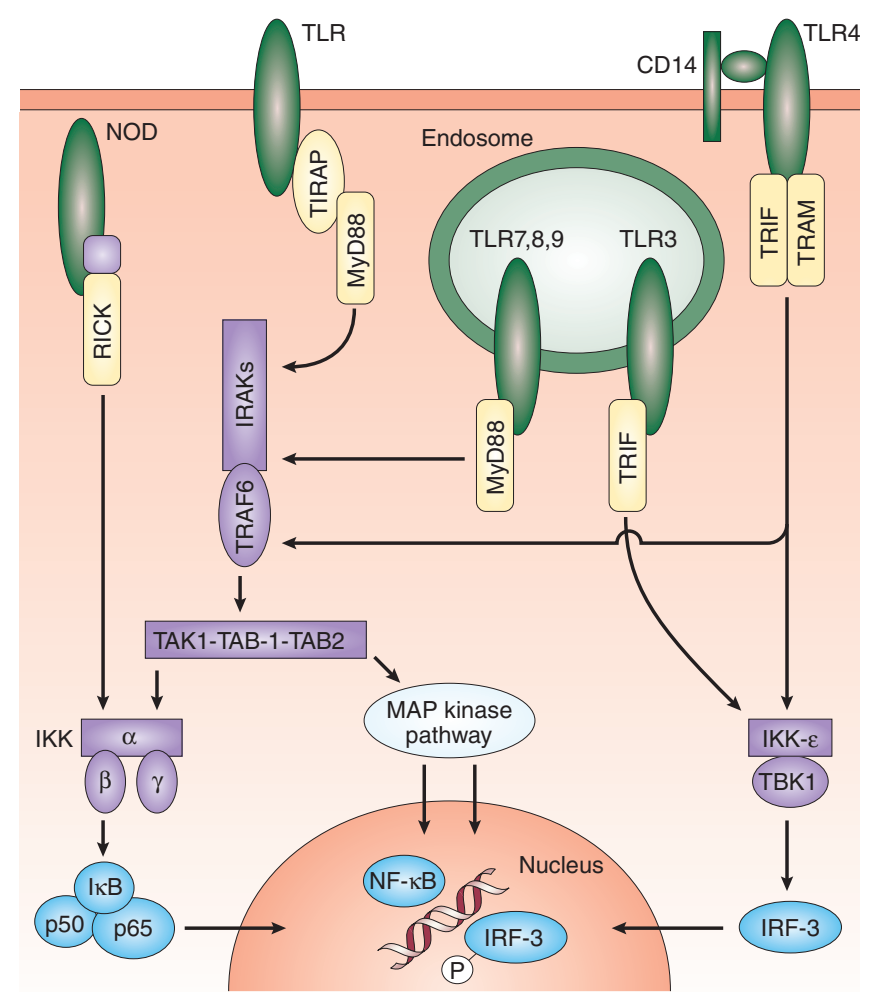

Figure 1 Signaling through the receptors of the innate immune system. Upon interaction with PAMPs, PRRs initiate signaling cascades leading to activation of transcription factors (such as NF-KB and IRF3), resulting in expression of inflammatory cytokines and other cellular activation events. A simplified pathway highlighting the main elements is shown, with receptors in green, adaptor proteins in yellow, kinases in purple and transcription factors in blue. Ligation of different TLRs may induce distinct gene expression patterns ${ }^{49}$. These newly identified receptors and signaling moieties of the innate immune system provide a rich source for the discovery of targeted immune potentiators and development as vaccine adjuvants. TRIF, TIR domain-containing adaptor protein inducing IFN- $\beta$; TRAM, TRIF-related adaptor molecules; TBK1, TRAF family member-associated $\mathrm{NF}-\kappa \mathrm{B}$ activator binding kinase 1 ; IKK, I $\mathrm{KB}$ kinase complex; TAK1, transforming growth factor $\beta$-activated kinase; TAB1/2, TAK1 binding protein. 
Figure 2 Initiation of the immune response. Upon interaction with PAMPs (1), resting innate immune cells and certain nonimmune cells, such as tissue epithelial cells, secrete cytokines and chemokines. Double arrows indicate cross-talk between cells. Key features of this early activation are an increase in APC function characterized by increased costimulatory capacity and upregulation of surface major histocompatibility complex molecules (2). Cells of the adaptive immune system, such as $\mathrm{CD} 4^{+}$and $\mathrm{CD} 8^{+} \mathrm{T}$ cells, then recognize presented antigen and undergo clonal expansion (3). Cognate interaction between $T$ and $B$ cells leads to $B$ cell activation, expansion and conversion to plasma cells that secrete specific antibodies (4). Activated dendritic cells can also act directly on $\mathrm{CD} 8^{+} \mathrm{T}$ cells to license them to become effector CTLs (5) and may also secrete soluble factors that block the immunosuppressive effects of $C D 25^{+} T_{\text {reg }}(6)$. A proportion of adaptive immune cells also differentiate into memory cells that will be ready for a secondary encounter with the specific pathogen. Thus, complete elimination of pathogens is achieved by effector cells and soluble factors of both the innate and adaptive immune systems.

Members of the NOD family of PRRs contain three distinct regions: a leucine-rich, pattern-recognition domain similar to TLRs; a nucleotidebinding domain that is essential for oligomerization and subsequent signaling; and effector motifs such as the caspase-recruitment domain. After association with their ligands, NOD1 and NOD2 activate NF- $\kappa B$ through a pathway that requires the interaction of receptor-interacting serine/threonine kinase (RICK) with the caspase-recruitment domain. Subsequent activation of the NF- $\kappa \mathrm{B}$ and MAPK pathways leads to the expression of numerous inflammatory cytokines and chemokines ${ }^{16,17}$. Thus, signaling of the innate immune system involves a complex and rapidly growing set of transmembrane and intracellular proteins that offer a wide range of potential targets for vaccine adjuvants (Fig. 1).

Role of innate immunity in conditioning the adaptive immunity PRR signaling results mainly in the activation of transcription factors such as NF- $\kappa \mathrm{B}$ and IRF3 (interferon regulatory factor 3), which provide the inflammatory context for the rapid activation of host defenses. The NF- $\kappa B$ pathway controls the expression of proinflammatory cytokines such as IL-1 $\beta$ and tumor necrosis factor- $\alpha$, whereas the IRF3 pathway leads to the production of antiviral type I interferons (IFN- $\alpha$ and IFN- $\beta)^{14}$. Along with these cytokines, various chemokines (such as IL-8, monocyte chemoattractant proteins, macrophage inflammatory proteins and RANTES) are released and their receptors are expressed on the surface of activated cells. As a result, vascular endothelial cells may alter surface expression of selectins and intercellular cell adhesion molecules, leading to the extravasation and selective retention of some leukocytes at the inflamed site. This cellular infiltrate consists of activated monocytes, neutrophils, basophils, eosinophils and natural killer cells, many of which also express TLRs and may subsequently become activated by the presence of their respective ligands. As a consequence of this inflammatory microenvironment, monocytes that infiltrate the site can differentiate into macrophages and dendritic cells. Thus, activation of the innate immune system can condition the inflamed site for the initiation of adaptive immune responses ${ }^{9}$.

Dendritic cells are recognized as one of the most important cell types for initiating the priming of naive $\mathrm{CD} 4^{+}$helper $\mathrm{T}\left(\mathrm{T}_{\mathrm{H}}\right)$ cells and for inducing $\mathrm{CD}^{+} \mathrm{T}$ cell differentiation into killer cells ${ }^{18}$. Immature dendritic cells are found at strategic anatomical sites throughout the body, thereby allowing them to respond rapidly to microbial invasion. Immature dendritic cells, on sensing potentially dangerous microbial signals through their PRRs, undergo maturation and subsequent migration to secondary lymphoid organs to prime naive T cells. At least three different subsets of dendritic cells (that is, those derived from myeloid, lymphoid and Langerhans cells) have been described based on their origins and phenotypic characteristics

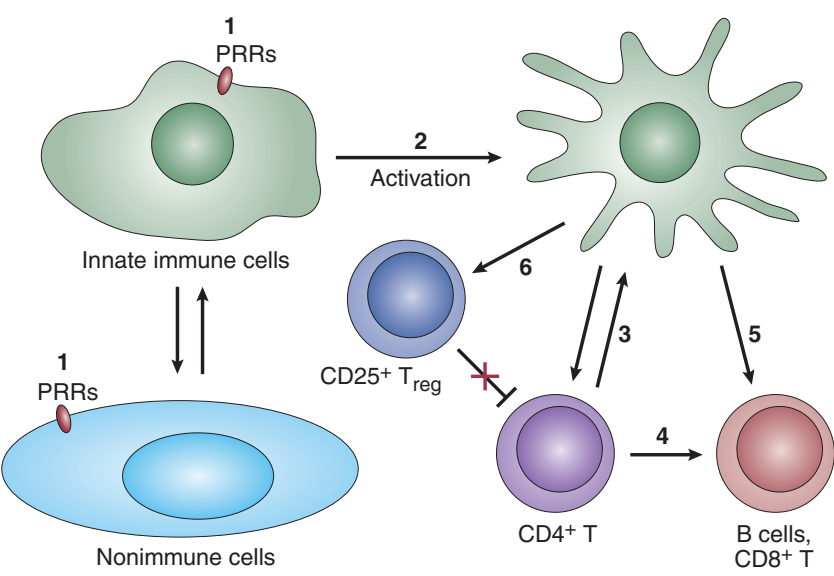

(including expression of distinct TLR repertoires) ${ }^{19}$. Hence, activation of different dendritic cells has the potential to induce qualitatively distinct immune responses. Activation of lymphoid dendritic cells, because of their preponderance to secrete IL-12, may be important for priming $\mathrm{T}_{\mathrm{H}} 1$-like responses, whereas early activation of myeloid dendritic cells may lead to $\mathrm{T}_{\mathrm{H}}$ 2- or $\mathrm{T}_{\mathrm{H}}$-like responses ${ }^{20}$. TLR signaling also has an important role in determining the quality of these helper $\mathrm{T}$ cell responses. For example, TLR2 engagement by its ligand Pam3Cys leads to a preferential production of $\mathrm{T}_{\mathrm{H}} 2$ cytokines, whereas lipopolysaccharide, which is a TLR4 ligand, leads to $\mathrm{T}_{\mathrm{H}} 1$ responses ${ }^{20,21}$. Dendritic cells may also exert indirect effects through, for example, the blockade of negative regulators of immune activation (Fig. 2). It has been suggested that the production of IL-6 by dendritic cells may be responsible for inhibiting the suppressor activity of $\mathrm{CD} 4^{+} \mathrm{CD} 25^{+}$ regulatory $\mathrm{T}$ cells $\left(\mathrm{T}_{\text {reg }}\right)^{22,23}$. In summary, various elements of the innate immune response are crucial for the generation of antigen-specific immunity and are thus key targets for vaccine adjuvants.

\section{Current state of adjuvants in vaccine research}

Potent adjuvants can improve the effectiveness of vaccines by accelerating the generation of robust immune responses, sustaining responses for a longer duration, inducing local mucosal immune responses, generating antibodies with increased avidity and neutralization capacity, eliciting cytotoxic T lymphocytes (CTLs), enhancing immune responses in individuals with weakened immune systems (for example, children, elderly or immunocompromised adults), increasing the response rate in low-responder individuals and reducing the amount of antigen needed, thus reducing the cost of vaccination programs. Adjuvants are functionally defined as components added to vaccine formulations that enhance the immunogenicity of antigens in vivo. Given our new understanding of innate immune mechanisms, this functional definition needs refinement and adjuvants now should be divided into two classes (delivery systems and immune potentiators) based on their dominant mechanisms of action. Immune potentiators activate innate immunity directly (for example, cytokines) or through PRRs (such as bacterial components), whereas delivery systems (for example, cationic microparticles ${ }^{24}$ ) may concentrate and display antigens in repetitive patterns, target vaccine antigens to APCs and help colocalize antigens and immune potentiators ${ }^{4}$. Thus, both immune potentiators and delivery systems can serve to augment antigen-specific immune response in vivo. For subunit vaccines it is probable that the combination of delivery systems, immune potentiators and isolated antigens will be required to elicit optimal immune responses.

Currently licensed adjuvants (Table 2) were developed using empirical methods, thus they are not optimal for many of the challenges in 
Table 2 Types of adjuvants and delivery systems ${ }^{a}$

\begin{tabular}{|c|c|c|}
\hline Adjuvant category & Representative examples & Brief description \\
\hline \multirow[t]{2}{*}{ Mineral salts } & \multirow{2}{*}{$\begin{array}{l}\text { Aluminum and calcium } \\
\text { salts }\end{array}$} & Licensed for human use \\
\hline & & $\begin{array}{l}\text { Many bacterial and viral } \\
\text { antigens have been adsorbed } \\
\text { onto alum and } \mathrm{Ca} \text { salts }\end{array}$ \\
\hline \multirow{2}{*}{$\begin{array}{l}\text { Emulsions and } \\
\text { surfactant-based } \\
\text { formulations }\end{array}$} & \multirow{2}{*}{$\begin{array}{l}\text { MF59, AS02, montanide } \\
\text { ISA-51 and ISA-720, } \\
\text { QS21 }\end{array}$} & $\begin{array}{l}\text { Micro-fluidized detergent- } \\
\text { stabilized emulsions }\end{array}$ \\
\hline & & $\begin{array}{l}\text { Surfactants derived from } \\
\text { natural sources }\end{array}$ \\
\hline
\end{tabular}

Particulate delivery Microparticles, immunovehicles stimulatory complexes; liposomes, virosomes, virus-like particles

Microbial deriva- Monophosphoryl lipid A, tives

\section{CpG oligonucleotides,} mimulators cholera toxin and heat labile the innate immune system toxin from Escherichia coli, lipoproteins

Antigens and adjuvants can be trapped inside or coated onto the surface of particles

Bacterial products or synthetic Most of these agents signal
Mnate immune system through TLRs

Cells and cytokines Dendritic cells; IL-12 and GM-CSF

Cytokines stimulate cells of the immune system
Autologous dendritic cells pulsed with tumor-derived peptides efficiently present antigenic epitopes

The main categories of vaccine adjuvants and delivery systems are summarized above. Most of these are still in experimental stages. The only ones licensed for human use are the aluminum salts, the oil-in-water emulsion MF59 and virosomes. For a recent review, see ref. 48. GM-CSF, granulocyte macrophage colony-stimulating factor.

vaccination today. In particular, the historical emphasis on humoral immune responses has led to the development of adjuvants with the ability to enhance antibody responses. As a consequence, most commonly used adjuvants are effective at elevating serum antibody titers, but do not elicit significant $\mathrm{T}_{\mathrm{H}} 1$ responses or CTLs ${ }^{25}$. The ability of an adjuvant to qualitatively affect the outcome of the immune response is an important consideration, because the need for vaccines against chronic infections (for example, human immunodeficiency virus (HIV), hepatitis $\mathrm{C}$ virus (HCV), tuberculosis and herpes simplex virus (HSV)) and cancer has shifted the focus to generation of cellular immune responses and adjuvants specifically geared toward eliciting this effect. To this end, many new and existing adjuvant formulations are being tested in various preclinical and clinical trials (Table 2). Our expanded understanding of the immunobiology of TLRs and other PRRs, immunoregulatory cells, dendritic cells and the importance of specific $T$ helper cell responses $\left(\mathrm{T}_{\mathrm{H}} 1\right.$ versus $\left.\mathrm{T}_{\mathrm{H}} 2\right)$ in resolving particular diseases provides a framework for their continued optimization.

\section{Immune potentiator discovery}

Much of the activity in vaccine development until now has been directed to finding new protective antigens and ways to present them, rather than to optimizing immune responses against them. As a result, only a few delivery systems (for example, aluminum salts and the oil in water emulsion MF59 (refs. 25-27); Table 2) and no immune potentiators have been approved for human use in prophylactic vaccines. This lack of progress in licensure of immune potentiators as adjuvants is the result of perceived toxicity risks and the lack of a comprehensive approach toward discovery of adjuvants ${ }^{28}$. Thus, to increase our portfolio of immune potentiator compounds to be used with new subunit vaccines and tailored to the specific requirements of each particular vaccine, a systematic approach to the discovery of new adjuvants is needed. A strategy that is used successfully in drug discovery is high-throughput screening ${ }^{29}$. For this approach to be applied to adjuvant discovery, there are two necessary requirements: diverse chemical libraries that contain compounds able to trigger innate immune responses, and in vitro assays that are predictive of in vivo adjuvant activity. The identification of the small, 'drug-like' imidazoquinoline family as TLR7- and TLR8-dependent immune potentiators suggests that modern drug discovery tools can be applied to vaccine adjuvant development ${ }^{30}$.

A general strategy of screening for immune potentiators is briefly outlined in Figure 3. An important early step in this process is the development of an assay amenable to high-throughput screening that measures a relevant immune parameter. One approach is to use cell-based screens based on immune activation that cast a broad net for immune potentiators in diverse chemical libraries. Alternatively, targeted screens for compounds that trigger PRR directly or through downstream signaling cascades can be established based on the growing understanding of these mechanisms. Regardless of which approach is used, novel compounds and their analogs identified in high-throughput screening will need to be optimized for further development. Again, drug discovery engines can be applied to the search for new immune potentiators. Hit to Lead (HTL) optimization is an iterative process of structural modification and creation of analogs followed by screening for improved activity to identify lead or optimized compounds. These lead compounds may then be tested for their efficacy in experimental vaccine formulations using established animal models and benchmarking against known immune potentiators such as $\mathrm{CpG}$ oligonucleotide sequences. The quantity and quality of immune responses can be judged by detection of functional antibodies, analysis of antigen-specific $\mathrm{CD} 4^{+}$and $\mathrm{CD} 8^{+} \mathrm{T}$ cells and protection against an infectious pathogen challenge.

\section{Functional screening}

A functional high-throughput screen without knowledge of intended targets can be carried out to test the ability of small molecular compounds to activate innate immune cells in vitro. These same assays can then be used for subsequent HTL optimization. This approach is based on the observation that all known immune potentiators stimulate immune cells in vitro and has several potential advantages. First, the methodology is straightforward; it relies on established immunological assays, such as enzyme-linked immunosorbent assay, to measure cytokines and/or chemokines produced by immune cell lines or primary cells (for example, human peripheral blood mononuclear cells). Second, the screen is unbiased and eliminates a priori assumptions about mode of action that may be premature given our rapidly evolving understanding of innate immune-triggering pathways. But significant downstream efforts will be required to identify the molecular targets of novel immune potentiators discovered in this way using cell-based assays. To this end, detailed phenotypic analyses of a response can be carried out using a variety of available genomics and proteomics approaches, which may aid in the identification of previously unexamined targets of immunological relevance.

\section{Targeted screening}

Various TLR ligands such as CpG DNA (TLR9) and imidazoquinolines (TLR7, TLR8) have been documented to stimulate cytokine production from immune cells in vitro. These compounds are being tested as vaccine adjuvants and immunotherapeutic agents for cancer and infectious disease, for which some efficacy has been shown ${ }^{31-33}$. The imidizoquinolines are particularly interesting because they are the first small, drug-like compounds shown to be TLR agonists ${ }^{30}$. Thus, libraries consisting of both natural compounds and their analogs (for example, nucleic acids) or synthetic small molecules could be screened using cells expressing specific TLRs and appropriate reporter genes to identify selective TLR agonists. Specifically, cotransfection of a single TLR cDNA with an $\mathrm{NF}-\kappa \mathrm{B}$ reporter construct into a TLR-deficient cell line may serve as a powerful screening tool for immune potentiators targeting that particu- 


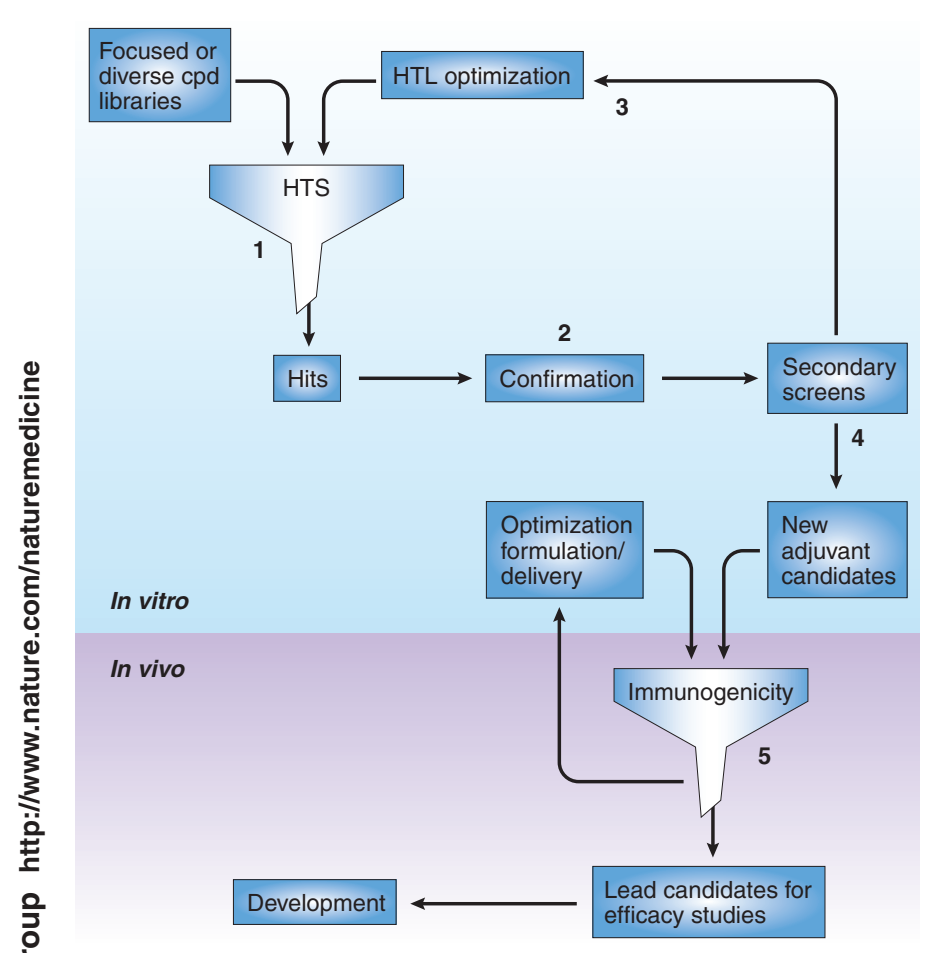

lar TLR. Other targeted approaches may involve the search for inhibitors of known immune antagonists (such as negative regulators of TLR signaling ${ }^{34}$ ) or compounds that potentiate positive regulators of the immune system ( such as activators of NF- $\kappa \mathrm{B}^{35}$ ). These biased approaches to adjuvant discovery may be most effective once the quality of the desired immune responses are known and the specific elements of the innate immune system involved in these responses are established.

\section{Search for novel peptide ligands of PRRs}

Most naturally occurring PAMPs are based on polysaccharides, lipids or nucleic acids (Table 1$)^{8}$. However, the peptide ALTTE, derived from bacterial fimbrae, was shown to activate TLR2 (refs. 36,37). Another peptide isolated from an experimentally infected insect was able to stimulate natural killer cells to produce IFN- $\gamma^{38}$. Thus, immunomodulators can also be based on peptides. Notably, the peptide sequence ALTTE is found in several human genes, thus peptide-based PAMPs are not necessarily limited to sequences of microbial origin. These observations suggest another dimension to immune potentiator discovery and open the possibility of screening peptide libraries generated by combinatorial peptide synthesis. However, barriers to the use of peptides as immune potentiators for vaccines are their poor pharmacokinetics and, in particular, their susceptibility to degradation in vivo, which may restrict their widespread use.

\section{Cell-based adjuvants and delivery systems}

Dendritic cells, as described earlier, are able to prime potent lymphocyte responses and are increasingly being tested for their ability to act as adjuvants in therapeutic vaccines against various cancers ${ }^{39-41}$. The labor-intensive nature of their preparation and the costs involved make cell-based vaccine adjuvants impractical in a prophylactic setting, but they are being evaluated for therapeutic vaccination. To effectively use dendritic cells in this way, they must be of the appropriate phenotype to optimally present antigenic peptides and express costimulatory molecules. Various TLR ligands, such as lipopolysaccharide and CpG DNA, have been tested for their capacity to condition ex vivo dendritic cells so that they express elevated levels of costimulatory molecules and surface
Figure 3 Rational approach to adjuvant discovery. Libraries of diverse chemical compounds (cpd) can be mined for immune potentiators using high-throughput screening (HTS) (1). Cell-based assays can be designed to measure general activation of innate immune cells or screens can be targeted to specific receptors or signaling components of the innate immune system such as TLRs. Active compounds (hits) identified in a primary screen are then confirmed and prioritized based on level of potency and selectivity (2). In HTL optimization, chemical analogs of the initial hits are generated and tested in an iterative fashion to identify compounds with increased potency and solubility (3). Secondary screens can be carried out to evaluate mechanism of action, toxicity and other parameters (4). The HTL-optimized immune potentiators (lead candidates) can then be formulated and tested for increased compatibility with different delivery systems. In vivo immunogenicity and toxicity studies using established vaccine candidates are conducted to identify lead immune potentiators (5), which then are put in development as novel vaccine adjuvants.

molecules associated with cell migration ${ }^{20}$. TLR-activated dendritic cells increase $\mathrm{T}$ cell responses and can block the inhibitory effects caused by $\mathrm{T}_{\text {reg }}$ cells $^{22}$. Moreover, mouse dendritic cells have been shown to aid migration and recruitment of natural killer cells to the lymph nodes to provide an early source of IFN- $\gamma$ necessary for $\mathrm{T}_{\mathrm{H}} 1$ polarization ${ }^{42}$. These insights may be modeled to develop functional or targeted highthroughput screening to identify synthetic compounds that optimally activate dendritic cells for use as cell-based adjuvants.

\section{Future perspectives}

Several forces are converging to drive increased research and development efforts in adjuvant design and discovery (Box 2) . First and foremost are the recent and dramatic breakthroughs in our theoretical and mechanistic understanding of innate immunity and how it drives antigen-specific responses and the generation of immunological memory. This new appreciation of innate defense mechanisms provides a solid foundation for rational approaches to immune potentiator discovery and optimization. Several first-generation candidates (for example, CpG, monophosphoryl lipid A and imidizoquinolines) have shown some efficacy in experimental animals and in phase 1 studies in humans ${ }^{31-33}$. Second, the trend in vac-

\section{BOX 2 CHALLENGES IN ADJUVANT DISCOVERY}

- Because of a need for qualitatively specific immune responses, no single adjuvant will be sufficient for broad applications. Thus, there may be a need for tailor-made adjuvants for specific vaccines.

- Available methods of delivery may be inadequate for small molecular adjuvants and therefore may require specialized formulations for delivery of small molecular compounds into cells and retention at the injection site.

- Potent activation of innate immune responses may lead to toxic side effects and this may necessitate delivery systems to limit diffusion from the injection site.

- Intracellular targets of immune potentiators may require development of membrane permeable and metabolically stable compounds.

- To fully exploit our recent insight into the innate immune system, expertise in various disciplines such as chemistry, biology, highthroughput instrumentation and formulation science will be needed for discovery, implementation and testing of new adjuvants.

- Regulatory guidelines on licensing of new adjuvants are still being formulated. Uncertainties remain regarding the scope of preclinical characterization and toxicity testing that will be required for approval. 
cine development away from traditional whole-cell or virus vaccines to subunit vaccines has shown that isolated antigens often lack sufficient immunogenicity, thus requiring the addition of potent adjuvants. Finally, the lack of vaccines for important disease targets such as HIV, HCV, HSV, Neisseria meningitides and others increases the need for improved vaccine adjuvants capable of boosting the antigen-specific immune response to protective levels against these insidious pathogens.

Although there is a growing acceptance by regulatory agencies and commercial vaccine producers that improved vaccine adjuvants are ๖ needed to meet the infectious disease challenges of the future, new technologies carry the uncertainty of unknown risks. At present, the safety and regulatory hurdles that will be encountered with the addition of novel immune potentiators and delivery systems to final vaccine formulations may be significant and are still largely ill defined. The key focus should be on separating the potential increases in immune toxicity from improved immunogenicity provided by vaccine adjuvants. Currently, our understanding of this trade-off is rudimentary but the tools and models are being developed to approach the question rationally (Fig. 3). It is likely that improved formulation and controlled release of potent immune potentiators will limit toxicities while increasing efficacy ${ }^{43}$. In addition, the growing number of immune potentiators, targeting diverse innate immune mechanisms, should allow for the identification of candidates with improved therapeutic indices. Thus, 으 the long-term goal should focus on selection of the optimal platforms and identification of key innate immune targets for induction of potent, but safe, immune responses. At this early stage, the hurdles seem high and public-private partnerships to fuel research and development may be required to overcome them. Such partnerships have already been established in the areas of HIV and biodefense vaccine research, and it is probable that government-industry cooperation on these particularly difficult vaccine problems will lead to breakthroughs in adjuvant development. The mechanistic understanding of the innate immune system and the tools to manipulate it are growing, and together these will make a significant impact on vaccine development in the near future.

\section{ACKNOWLEDGMENTS}

We gratefully acknowledge N. Cronen for preparation of illustrations.

\section{COMPETING INTERESTS STATEMENT}

The authors declare competing interests (see the Nature Medicine website for details).

Published online at http://www.nature.com/naturemedicine/

1. Hilleman, M.R. Vaccines in historic evolution and perspective: a narrative of vaccine discoveries. Vaccine 18, 1436-1447 (2000).

2. Kieny, M.P., Excler, J.L. \& Girard, M. Research and development of new vaccines against infectious diseases. Am. J. Public Health 94, 1931-1935 (2004).

3. Hoebe, K., Janssen, E. \& Beutler, B. The interface between innate and adaptive immunity. Nat. Immunol. 5, 971-974 (2004).

4. O'Hagan, D.T. Recent developments in vaccine delivery systems. in New Generation Vaccines 2 nd edn. (eds. Levine, M.M., Kaper, J.B., Rappuoli, R., Liu, M. \& Good, M.F.) 259-270 (Marcel Dekker, New York, 2004).

5. Medzhitov, R. \& Janeway, C.A., Jr. Innate immunity: the virtues of a nonclonal system of recognition. Cell 91, 295-298 (1997).

6. Gourley, T.S., Wherry, E.J., Masopust, D. \& Ahmed, R. Generation and maintenance of immunological memory. Semin. Immunol. 16, 323-333 (2004).

7. Janeway, C.A. Jr. Approaching the asymptote? Evolution and revolution in immunology. Cold Spring Harbor Symp. Quant. Biol. 54, 1-13 (1989).

8. Barton, G.M. \& Medzhitov, R. Toll-like receptors and their ligands. Curr. Top. Microbiol. Immunol. 270, 81-92 (2002).

9. Iwasaki, A. \& Medzhitov, R. Toll-like receptor control of the adaptive immune responses. Nat. Immunol. 5, 987-995 (2004).

10. Gordon, S. Pattern recognition receptors: doubling up for the innate immune response. Cell 111, 927-930 (2002).

11. Sato, M. et al. Direct binding of Toll-like receptor 2 to zymosan, and zymosaninduced NF- $\mathrm{KB}$ activation and TNF- $\alpha$ secretion are down-regulated by lung collectin surfactant protein A. J. Immunol. 171, 417-425 (2003).

12. Lien, E. et al. Toll-like receptor 4 imparts ligand-specific recognition of bacterial lipopolysaccharide. J. Clin. Invest. 105, 497-504 (2000).
13. Rutz, M. et al. Toll-like receptor 9 binds single-stranded CpG-DNA in a sequence- and pH-dependent manner. Eur. J. Immunol. 34, 2541-2550 (2004).

14. Akira, S. \& Takeda, K. Toll-like receptor signalling. Nat. Rev. Immunol. 4, 499-511 (2004).

15. Akira, S., Yamamoto, M. \& Takeda, K. Role of adapters in Toll-like receptor signalling. Biochem. Soc. Trans. 31, 637-642 (2003).

16. Chamaillard, M. et al. An essential role for NOD1 in host recognition of bacterial peptidoglycan containing diaminopimelic acid. Nat. Immunol. 4, 702-707 (2003).

17. Girardin, S.E. et al. Nod2 is a general sensor of peptidoglycan through muramyl dipeptide (MDP) detection. J. Biol. Chem. 278, 8869-8872 (2003).

18. Banchereau, J. et al. Immunobiology of dendritic cells. Annu. Rev. Immunol. 18, 767811 (2000).

19. Shortman, K. \& Liu, Y.J. Mouse and human dendritic cell subtypes. Nat. Rev. Immunol. 2, 151-161 (2002).

20. Pulendran, B. Modulating vaccine responses with dendritic cells and Toll-like receptors. Immunol. Rev. 199, 227-250 (2004).

21. Dabbagh, K. \& Lewis, D.B. Toll-like receptors and T-helper-1/T-helper-2 responses. Curr. Opin. Infect. Dis. 16, 199-204 (2003).

22. Pasare, C. \& Medzhitov, R. Toll pathway-dependent blockade of $\mathrm{CD} 4^{+} \mathrm{CD} 25^{+} \mathrm{T}$ cellmediated suppression by dendritic cells. Science 299, 1033-1036 (2003).

23. Pasare, C. \& Medzhitov, R. Toll-dependent control mechanisms of CD4 T cell activation. Immunity 21, 733-741 (2004).

24. O'Hagan, D.T. et al. Cationic microparticles are a potent delivery system for a HCV DNA vaccine. Vaccine $\mathbf{2 3}, 672-680$ (2004).

25. Lindblad, E.B. Aluminium adjuvants-in retrospect and prospect. Vaccine 22, 36583668 (2004).

26. Lindblad, E.B. Aluminium compounds for use in vaccines. Immunol. Cell Biol. 82, 497505 (2004).

27. Ott, G. The Adjuvant MF59: A ten year perspective. in Vaccine Adjuvants; Preparation Methods and Research Protocols (ed. O'Hagan, D.) pp. 211-228 (Humana Press, Totowa, New Jersey, USA, 2001).

28. Gupta, R.K. \& Siber, G.R. Adjuvants for human vaccines - current status, problems and future prospects. Vaccine 13, 1263-1276 (1995).

29. Liu, B., Li, S. \& Hu, J. Technological advances in high-throughput screening. Am. J. Pharmacogenomics 4, 263-276 (2004).

30. Hemmi, H. et al. Small anti-viral compounds activate immune cells via the TLR7 MyD88-dependent signaling pathway. Nat. Immunol. 3, 196-200 (2002).

31. Cooper, C.L. et al. Safety and immunogenicity of CPG 7909 injection as an adjuvant to Fluarix influenza vaccine. Vaccine 22, 3136-3143 (2004).

32. Mason, K.A. et al. Targeting Toll-like receptor 9 with CpG oligodeoxynucleotides enhances tumor response to fractionated radiotherapy. Clin. Cancer Res. 11, 361-369 (2005).

33. Hengge, U.R. \& Cusini, M. Topical immunomodulators for the treatment of external genital warts, cutaneous warts and molluscum contagiosum. Br. J. Dermatol. 149 (suppl. 66), 15-19 (2003).

34. Fukao, T. \& Koyasu, S. PI3K and negative regulation of TLR signaling. Trends Immunol. 24, 358-363 (2003).

35. Karin, M., Yamamoto, Y. \& Wang, Q.M. The IKK NF-kB system: a treasure trove for drug development. Nat. Rev. Drug Discov. 3, 17-26 (2004).

36. Asai, Y., Ohyama, Y., Gen, K. \& Ogawa, T. Bacterial fimbriae and their peptides activate human gingival epithelial cells through Toll-like receptor 2. Infect. Immun. 69, 7387-7395 (2001).

37. Ogawa, T., Asai, Y., Hashimoto, M. \& Uchida, H. Bacterial fimbriae activate human peripheral blood monocytes utilizing TLR2, CD14 and CD11a/CD18 as cellular receptors. Eur. J. Immunol. 32, 2543-2550 (2002).

38. Chernysh, S. et al. Antiviral and antitumor peptides from insects. Proc. Natl. Acad. Sci. USA 99, 12628-12632 (2002).

39. Zitvogel, L. et al. IL-12-engineered dendritic cells serve as effective tumor vaccine adjuvants in vivo. Ann. NY Acad. Sci. 795, 284-293 (1996).

40. Grohmann, U. et al. Dendritic cells and interleukin 12 as adjuvants for tumor specific vaccines. Adv. Exp. Med. Biol. 417, 579-582 (1997).

41. Mayordomo, J.I. et al. Bone marrow-derived dendritic cells serve as potent adjuvants for peptide-based antitumor vaccines. Stem Cells 15, 94-103 (1997).

42. Martin-Fontecha, A. et al. Induced recruitment of NK cells to lymph nodes provides IFN- $\gamma$ for $\mathrm{T}_{H} 1$ priming. Nat. Immunol. 5, 1260-1265 (2004).

43. O'Hagan, D.T. \& Valiante, N.M. Recent advances in the discovery and delivery of vaccines and adjuvants. Nat. Rev. Drug Discov. 2, 727-735 (2003).

44. Nakao, Y. et al. Surface-expressed TLR6 participates in the recognition of diacylated lipopeptide and peptidoglycan in human cells. J. Immunol. 174, 15661573 (2005).

45. Sandor, F. et al. Importance of extra- and intracellular domains of TLR1 and TLR2 in NFKB signaling. J. Cell Biol. 162, 1099-1110 (2003).

46. Jurk, M. et al. Human TLR7 or TLR8 independently confer responsiveness to the antiviral compound R-848. Nat. Immunol. 3, 499 (2002).

47. Roberts, T.L., Sweet, M.J., Hume, D.A. \& Stacey, K.J. Cutting edge: species-specific TLR9-mediated recognition of $\mathrm{CpG}$ and non-CpG phosphorothioate-modified oligonucleotides. J. Immunol. 174, 605-608 (2005).

48. Pink, J.R. \& Kieny, M.P. 4th Meeting on Novel Adjuvants Currently In/Close to Human Clinical Testing, World Health Organization-Organisation Mondiale de la Sante, Fondation Merieux, Annecy, France, 23-25, June 2003. Vaccine 22, 2097-2102 (2004).

49. Dillon, S. et al. A Toll-like receptor 2 ligand stimulates Th2 responses in vivo, via induction of extracellular signal-regulated kinase mitogen-activated protein kinase and c-Fos in dendritic cells. J. Immunol. 172, 4733-4743 (2004). 